\title{
Method for determining the transmission of a garret window from a direct sun component
}

\author{
Khazret Guketlov*, and Islam Tatarov
}

Kabardino-Balkarian State University named after Kh.M. Berbekov, 360004, Nalchik, Russia

\begin{abstract}
Based on the experimental studies using the method of physical modeling under an artificial horizon NIISPh Moscow and the method of mathematical planning of the experiment, an analytical dependence of the light transmission coefficient on the direct component of sunlight, which characterizes the relationship between the height of the sun, geometric and lighting parameters of point skylights, was obtained.
\end{abstract}

\section{Introduction}

Unlike a cloudy sky, with a clear sky, the luminous flux incident on the entrance cavity of the light opening consists of two components: the flux coming from the sky (the scattered component) $F^{s}$ and the stream coming from the sun (direct component) $F^{\Theta}$ (Figure 1). The total luminous flux entering the room is determined by the formula

$$
F_{\text {outp }}=F^{s} \tau^{\text {c.s. }}+F^{\Theta} \tau^{\Theta}
$$

where $\tau^{\text {c.s. }}$ is the coefficient of light transmission from the scattered component of the luminous flux, is determined by $[1,2] ; \tau^{\Theta} \mathrm{s}$ the coefficient of light transmission from the direct component of the luminous flux.

Given the complexity of the redistribution of the luminous flux from the direct component, when passing through the opening of the garret window, the determination of the light transmission coefficient was carried out on the basis of the experimental research, at the working site of the installation of a small "Artificial horizon" NIISPH.

\footnotetext{
*Corresponding author: 123guket@mail.ru
} 


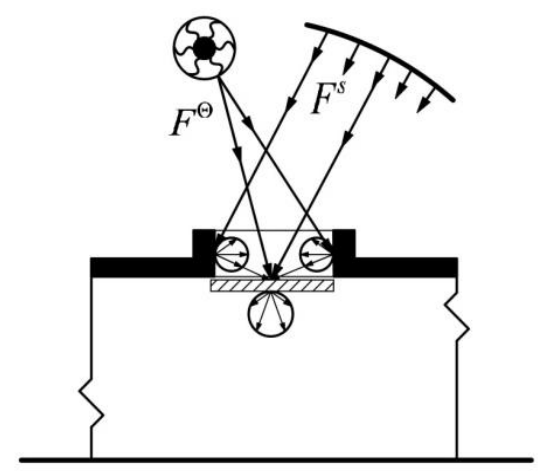

Fig. 1. Scheme of the light fluxes passage through an garret window in a clear sky

The experimental setup (Fig. 2) consisted of a searchlight moving across the sky - 5 searchlights with a parabolic mirror - 3, a tube-cylinder - 7, a box - 2 of 1 x 1 x $1 \mathrm{~m}$ size, the inner surface of which was evenly coated with white paint with a reflectivity $\rho=0,85$ and scattering light according to Lambert's law. Two holes were cut in the box lid. A model of a light opening or a diaphragm - 4 was installed in a large hole, in another - a photocell 1 with a screen -8 . The photocell was connected to a galvanometer -6 .

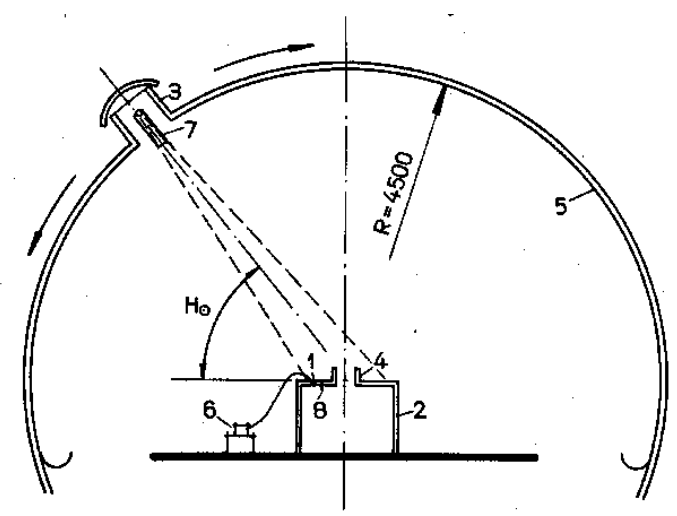

Fig. 2. Diagram of an experimental setup for measuring the light transmission coefficient for a garret window.

The installation worked on the principle of a photometric sphere. Light transmission coefficient of the light of the garret window $\tau^{\Theta}$ was determined from the ratio of the galvanometer indicators recording the steady-state illumination $E_{1}$ after the passage of the luminous flux through the skylight opening, to the indication of the galvanometer $\mathrm{n}_{2}$, recording steady-state illumination $E_{2}$ after passing the light flux through the diaphragm.

$$
\tau^{\Theta}=n_{1} / n_{2}
$$

To display the functional dependence of the light transmission coefficient, which characterizes the relationship between the height of the sun $\mathrm{H}^{\Theta}$, index of the skylight of the garret window $\mathrm{i}$ and the reflection coefficient of the garret window walls $\rho$ the method of mathematical planning of the experiment was applied [3]. The measurements 
were carried out according to the D-optimal design for three independent variables,

$$
i=\frac{a b}{h(a+b)}
$$

where $h$ is the height of the clear opening of the anti-aircraft spotlight; ${ }^{a, b}$ are the garret window dimensions in plan.

Range of the first controlled factor change $x_{1}=\mathrm{i}$ was within $0,5 \leq \mathrm{i} \leq 6$ and covered the geometric proportions of all unified garret windows.

Range of the second controlled factor variation $x_{2}=\rho$ was determined by the practical feasibility of finishing the walls of the garret window opening $0,4 \leq \rho \leq 0,8$.

Variation range of the third controlled factor $x_{3}=\mathrm{H}^{\Theta}$ covered the possible heights of the Sun $10 \leq \mathrm{H}^{\Theta} \leq 80$

As shown by the preliminary calculations, the range of the first controlled factor variation $x_{1}=\mathrm{i}$ does not allow to describe the process under study with one equation adequately. Therefore, the intervals of the first controlled factor were changed twice

a) $0.5 \leq \mathrm{i} \leq 2$,

b) $2 \leq i \leq 6$.

In accordance with the adopted D-optimal plan for the three-factor process study, a rectangular matrix of the experiment was compiled for I and II plans for 14 experiments, which is presented in Table 1.

Table 1. Matrix for the experimental plans I and II

\begin{tabular}{|c|r|r|c|c|c|c|c|}
\hline \multirow{2}{*}{$\begin{array}{c}\text { Experiment } \\
\text { no. }\end{array}$} & \multicolumn{3}{|c|}{ Experiment plan } & \multicolumn{5}{c|}{ Controllable factors } \\
\cline { 2 - 8 } & $\mathrm{x}_{1}$ & $\mathrm{x}_{2}$ & $\mathrm{x}_{3}$ & $\rho$ & $\mathrm{H}^{\Theta}$ & $0.5 \leq \mathrm{i} \leq 2$ & $2 \leq i \leq 6$ \\
\hline 1 & +1 & +1 & +1 & 0.8 & 80 & 2 & 6 \\
\hline 2 & -1 & -1 & +1 & 0.4 & 10 & 2 & 6 \\
\hline 3 & +1 & +1 & -1 & 0.8 & 80 & 0.5 & 2 \\
\hline 4 & -1 & +1 & +1 & 0.4 & 80 & 2 & 6 \\
\hline 5 & -1 & -1 & -1 & 0.4 & 10 & 0.5 & 2 \\
\hline 6 & +1 & -1 & +1 & 0.8 & 10 & 2 & 6 \\
\hline 7 & +1 & -1 & -1 & 0.8 & 10 & 0.5 & 2 \\
\hline 8 & -1 & +1 & -1 & 0.4 & 80 & 0.5 & 2 \\
\hline 9 & 0 & 0 & -1 & 0.6 & 45 & 0.5 & 2 \\
\hline 10 & 0 & 0 & +1 & 0.6 & 45 & 2 & 6 \\
\hline 11 & -1 & 0 & 0 & 0.4 & 45 & 1.25 & 4 \\
\hline 12 & +1 & 0 & 0 & 0.8 & 45 & 1.25 & 4 \\
\hline 13 & 0 & +1 & 0 & 0.6 & 80 & 1.25 & 4 \\
\hline 14 & 0 & -1 & 0 & 0.6 & 10 & 1.25 & 4 \\
\hline
\end{tabular}

The controllable factors were normalized by linear transformations in the matrices of the plans for the experiments. The transition from real values to normalized variables is made according to the formula. 


$$
x_{\mathrm{i}}=\frac{z_{i}-\frac{b_{i}+a_{i}}{2}}{\frac{b_{i}-a_{i}}{2}}
$$

where $a_{i}, b_{i}$ determine the range of variation of the studied variables $z_{i}\left(i, \rho, \mathrm{H}^{\Theta}\right)$, belonging to the domain of the input factors changing task.

The results of the experimental studies are presented in Table 2.

Table 2. Matrix and experimental results

\begin{tabular}{|c|c|c|c|c|c|}
\hline \multirow{2}{*}{$\begin{array}{c}\text { Experiment } \\
\text { no. }\end{array}$} & \multicolumn{3}{|c|}{ Experiment plan } & \multirow{2}{*}{$\begin{array}{l}\text { Averaged } \\
\text { function value } \mathrm{Y}_{\mathrm{i}} \\
\text { for } \\
\quad 0.5 \leq \mathrm{i} \leq 2\end{array}$} & \multirow{2}{*}{$\begin{array}{l}\text { Averaged } \\
\text { function value } \\
\mathrm{Y}_{\mathrm{i}} \text { for } \\
\quad 2 \leq i \leq 6\end{array}$} \\
\hline & $\mathrm{X}_{1}$ & $\mathrm{x}_{2}$ & $\mathrm{X}_{3}$ & & \\
\hline 1 & +1 & +1 & +1 & 0.96 & 0.98 \\
\hline 2 & -1 & -1 & +1 & 0.56 & 0.69 \\
\hline 3 & +1 & +1 & -1 & 0.84 & 0.95 \\
\hline 4 & -1 & +1 & +1 & 0.82 & 0.96 \\
\hline 5 & -1 & -1 & -1 & 0.04 & 0.52 \\
\hline 6 & +1 & -1 & +1 & 0.78 & 0.9 \\
\hline 7 & +1 & -1 & -1 & 0.3 & 0.78 \\
\hline 8 & -1 & +1 & -1 & 0.63 & 0.84 \\
\hline 9 & 0 & 0 & -1 & 0.32 & 0.79 \\
\hline 10 & 0 & 0 & +1 & 0.79 & 0.87 \\
\hline 11 & -1 & 0 & 0 & 0.58 & 0.79 \\
\hline 12 & +1 & 0 & 0 & 0.81 & 0.93 \\
\hline 13 & 0 & +1 & 0 & 0.86 & 0.95 \\
\hline 14 & 0 & -1 & 0 & 0.4 & 0.77 \\
\hline
\end{tabular}

The application correctness of the statistical estimates for processing the results obtained was carried out using $\tau$ distribution. The test for reproducibility of the studied process was carried out according to the Cochran criterion. The construction of mathematical models was carried out by the least squares method. The significance of the regression equations' obtained coefficients was checked according to the Student's criterion, the adequacy of the obtained mathematical models and results - according to Fisher's criterion with a confidence level of $95 \%$.

After the implementation of the plans for the experiments, the analytical expressions to determine the coefficient of light transmission of a point garret window from the direct component of the Sun $\tau^{\Theta}$, were obtained; they are represented by a regression equation of the form.

For the experimental plan I $0.5 \leq i \leq 2$

$$
\tau^{\Theta}=-0.14+0.759 i-1.172 \rho+0.112 H^{\Theta}-0.142 i^{2}+1.5 \rho^{2}-0.0034 i H^{\Theta}-0.003 \rho H^{\Theta},
$$

For the experimental plan II $2 \leq i \leq 6$

$$
\begin{aligned}
& \tau^{\Theta}=-0.0329+0.1255 i+0.8182 \rho+0.00755 H^{\Theta}-0.00755 i^{2}-0.04375 \rho i- \\
& -0.0003 i H^{\Theta}-0.00607 \rho H^{\Theta}
\end{aligned}
$$


Fig. 3,4,5 shows the change in the light transmission coefficient of the skylight for direct sunlight $\tau^{\Theta}$, with the reflection coefficient of the side faces $\rho=0.4,0.6$ and 0.8

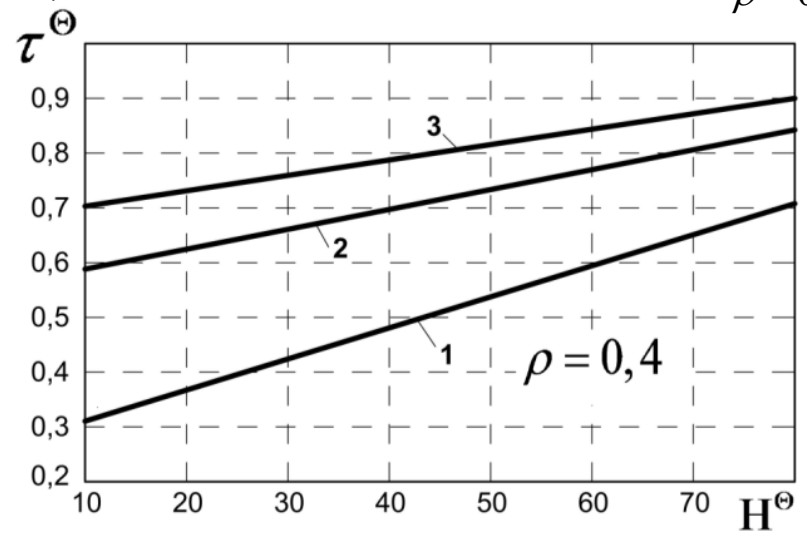

Fig. 3. Dependence of the light transmission coefficient of a spotted skylight on the sun height: $1-$ $i=1 ; 2-i=3 ; 3-i=6$ at $\rho=0.4$

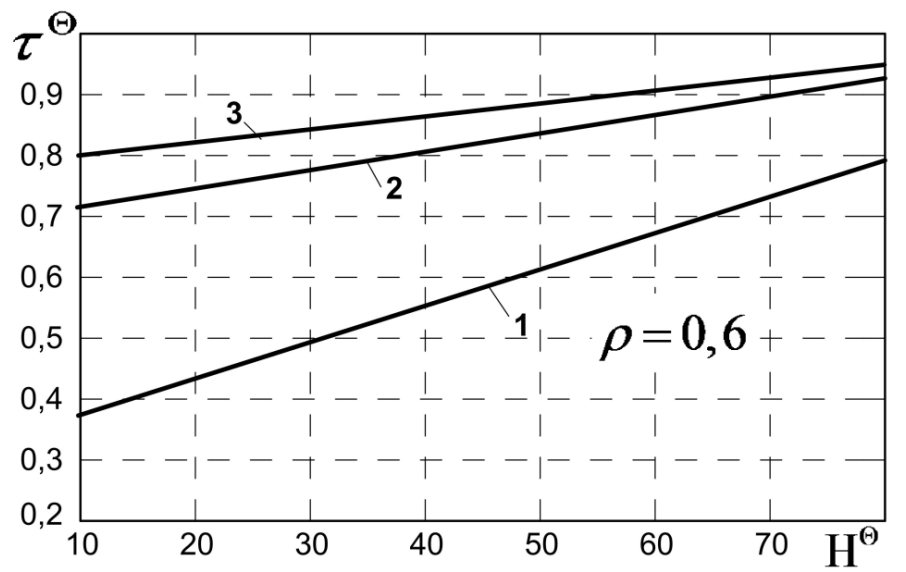

Fig. 4. Dependence of the light transmission coefficient of a point skylight on the sun height: 1 $i=1 ; 2-i=3 ; 3-i=6$ at $\rho=0.6$

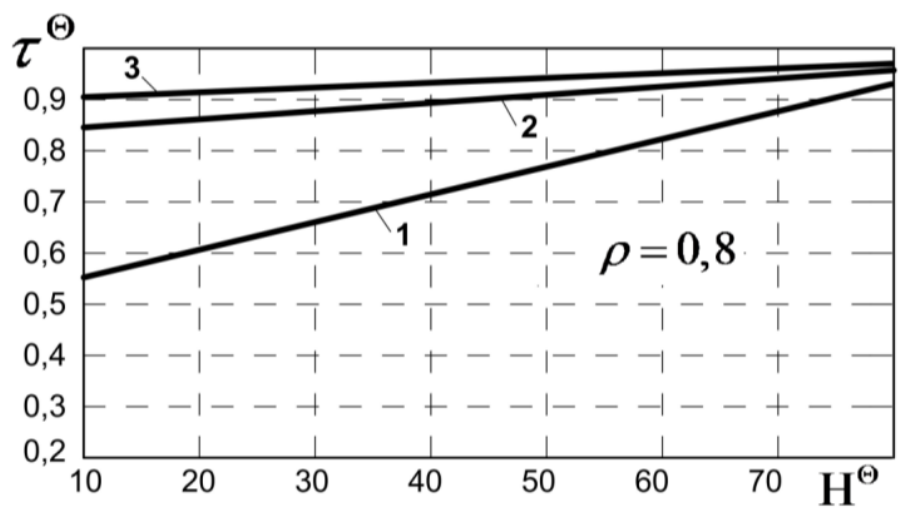

Fig. 5. Dependence of the light transmission coefficient of a spotted skylight on the sun height: 1 $i=1 ; 2-i=3 ; 3-i=6$ at $\rho=0.8$ 


\section{Conclusion}

The proposed method will make it possible, to more fully take into account the natural light energy resources of the construction site, when calculating natural illumination in spaces with garret windows which is important in the transition from the average accounting of the light climate to a differentiated one.

\section{References}

1. Kh.M. Guketlov, The method of differentiated accounting for the light climate in the calculation and design of the upper natural lighting of industrial buildings, Abstract of PhD thesis, (NIISPh, M., 1985)

2. Kh. M. Guketlov *, Kh. A. Khachetlov, K. A. Kibishev. IOP Conf. Series: Materials Science and Engineering 913, 042001 (2020)

3. Yu.N. Berezhnev, L.P. Derevianko, Recommendations for planning the experimental studies of mining machines (DONNIGRI Publishing House, Donetsk, 1975) 